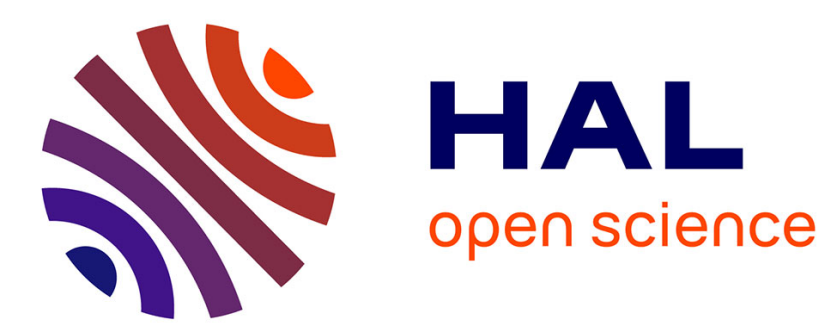

\title{
INVERSION IS POSSIBLE IN GROUPS WITH NO PERIODIC AUTOMORPHISMS
}

\author{
Martin R. Bridson, Hamish Short
}

\section{To cite this version:}

Martin R. Bridson, Hamish Short. INVERSION IS POSSIBLE IN GROUPS WITH NO PERIODIC AUTOMORPHISMS. Proceedings of the Edinburgh Mathematical Society, 2016, 10.1017/S0013091514000388 . hal-01261851

\section{HAL Id: hal-01261851 \\ https://hal.science/hal-01261851}

Submitted on 25 Jan 2016

HAL is a multi-disciplinary open access archive for the deposit and dissemination of scientific research documents, whether they are published or not. The documents may come from teaching and research institutions in France or abroad, or from public or private research centers.
L'archive ouverte pluridisciplinaire HAL, est destinée au dépôt et à la diffusion de documents scientifiques de niveau recherche, publiés ou non, émanant des établissements d'enseignement et de recherche français ou étrangers, des laboratoires publics ou privés. 


\title{
INVERSION IS POSSIBLE IN GROUPS WITH NO PERIODIC AUTOMORPHISMS
}

\author{
MARTIN R. BRIDSON AND HAMISH SHORT
}

\begin{abstract}
There exist infinite, finitely presented, torsion-free groups $G$ such that $\operatorname{Aut}(G)$ and $\operatorname{Out}(G)$ are torsion-free but $G$ has an automorphism sending some non-trivial element to its inverse.
\end{abstract}

\section{INTRODUCTION}

Several authors have investigated the nature of finite groups that do not admit an automorphism sending any non-trivial element to its inverse. H. Heineken and H. Liebeck [7] introduced the term NI ("no inversions") to describe such groups. If a finite group $G$ has an automorphism sending an element of order greater than 2 to its inverse, then $G$ has an automorphism of order 2. P. Hegarty and D. MacHale [6] enquired about the corresponding statement for infinite groups.

Theorem A. There exists an infinite, torsion-free, finitely presented group $G$ such that $\operatorname{Aut}(G)$ and $\operatorname{Out}(G)$ are torsion-free but there exist $\alpha \in \operatorname{Aut}(G)$ and $\gamma \in G \backslash\{1\}$ such that $\alpha(\gamma)=\gamma^{-1}$. Moreover, $G$ is co-Hopfian and has a finite classifying space.

A group $G$ is said to be co-Hopfian if every monomorphism $G \rightarrow G$ is an isomorphism.

We shall assume that the reader is familiar with Gromov's theory of hyperbolic groups, as described in [5], [1] and [3] Chapter III.H. A finitely generated subgroup $\Lambda$ of a hyperbolic $H$ is quasiconvex if the inclusion map $\Lambda \hookrightarrow H$ is a quasi-isometric embedding (with respect to any choice of word metrics). Quasiconvex free subgroups abound in hyperbolic groups. Indeed, if $H$ is hyperbolic and torsion-free, then for any $a, b \in H$ and all sufficiently large integers $n$, the subgroup $\left\langle a^{n}, b^{n}\right\rangle$ is free and quasiconvex. One says that a group $G$ splits over a free group if it can be expressed as an amalgamated free product or HNN extension with free amalgamated subgroups.

We shall deduce Theorem A from the following extension theorem for complete groups. Recall that a group $G$ is termed complete (or

1991 Mathematics Subject Classification. 20E36, 20F67.

Key words and phrases. Group automorphisms, hyperbolic groups, complete groups.

Bridson was supported in part by grants from the EPSRC and a Wolfson Merit Award from the Royal Society. 
asymmetric) if it has trivial centre and all of its automorphisms are inner; so $G \cong \operatorname{Inn}(G) \cong \operatorname{Aut}(G)$.

Theorem B. Let $H$ be a complete, torsion-free, hyperbolic group, let $F<H$ be a quasiconvex subgroup that is free of rank two, let $\phi: F \rightarrow F$ be an automorphism that has infinite order in $\operatorname{Out}(F)$, and let $G=H *_{\phi}$ be the corresponding $H N N$ extension. If $H$ does not split over any free group, then $G$ is torsion-free, complete, co-Hopfian, and has a finite classifying space.

This theorem would be of little use if we were unable to exhibit groups $H$ with the stated properties. To this end, note that by Mostow rigidity, if $M$ is a closed oreintable hyperbolic manifold of dimension $n \geq 3$, then $\pi_{1} M$ is asymmetric if and only if $M$ is asymmetric, i.e. $M$ has no non-trivial isometries. If $\pi=\pi_{1} M$ splits non-trivially as an amalgamated free product, say $\pi \cong A *_{C} B$, then there is a MayerVietoris exact sequence for integral homology groups

$$
\cdots \rightarrow H_{n} A \oplus H_{n} B \rightarrow H_{n} \pi \rightarrow H_{n-1} C \rightarrow \cdots
$$

By Poincaré duality, $H_{n} \pi \cong \mathbb{Z}$ and (since $A, B<\pi$ are of infinite index) $H_{n} A=H_{n} B=0$. Therefore $H_{n-1} C$ is infinite. In particular, since $n \geq 3$, the group $C$ cannot be free. A similar argument shows that $\pi$ does not split as an HNN extension over a free group either.

Thus $\pi_{1} M$ will have the properties that we require of $H$ if we can find a closed asymmetric hyperbolic manifold $M$ in some dimension $n \geq 3$. In fact, there exist such manifolds in every dimension $n \geq 3$ : Kojima [8] constructed examples in dimension 3 and, inspired by arguments of Long and Reid [9], Belolipetsky and Lubotzky [2] constructed infinitely many examples in each dimension $n \geq 3$.

With suitable groups $H$ in hand, it is easy to deduce Theorem A from Theorem B: if $\phi: F \rightarrow F$ has infinite order in $\operatorname{Out}(F)$ and sends some element $\gamma \in F \backslash\{1\}$ to its inverse, then it suffices to declare $\alpha: G \rightarrow G$ to be conjugation by the stable letter of $G=H *_{\phi}$. To obtain concrete examples, one can take $\phi$ to be the automorphism of Free $(a, b)$ given by $a \mapsto a^{-1}, b \mapsto b^{-1} a^{n}$.

\section{The Main Argument}

Our first aim is to prove that the centralizer $C_{G}(F)$ of the quasiconvex subgroup $F$ in the statement of Theorem $\mathrm{B}$ is trivial. For this, we need four lemmas.

Lemma 1. Let $A$ be a torsion-free group and let $F<A$ be a free subgroup of rank two. If $|A: F|$ is finite, then $A=F$.

Proof. Torsion-free virtually free groups are free, and a proper subgroup of finite-index in a finitely generated non-abelian free group has rank greater than that of the ambient group. 


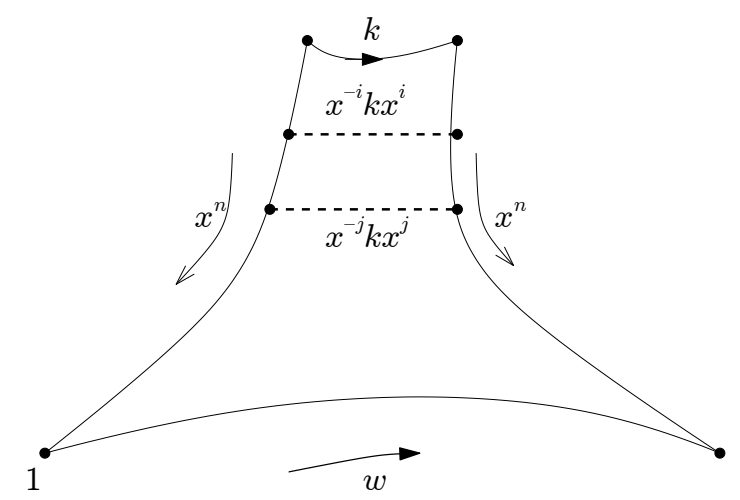

Lemma 2. Let $H$ be a torsion-free group that does not contain a copy of $\mathbb{Z}^{2}$. If $F<H$ is free of rank two, then $N_{H}(F)=F$.

Proof. Let $t \in N_{H}(F)$, the normalizer of $F$ in $H$. Every automorphism of $F=\langle a, b\rangle$ sends $c=[a, b]$ to a conjugate of $c^{ \pm 1}$ (see for instance [10, page 165]). Thus $t^{2} c t^{-2}=x c x^{-1}$ for some $x \in F$. If $t$ has finite order in $N_{H}(F) / F$, then $F$ has finite index in $A=\langle F, t\rangle$ and by Lemma 1 , $t \in F=A$. If $t$ has infinite order in $N_{H}(F) / F$, then $\langle F, t\rangle$ contains $\left\langle t^{-2} x, c\right\rangle \cong \mathbb{Z}^{2}$, contrary to hypothesis.

The following result is due to Mihalik and Towle [11]. We include a proof for the sake of completeness.

Lemma 3. Let $H$ be a torsion-free hyperbolic group. If $K<H$ is quasiconvex, then there does not exist an element $x \in H$ such that $x^{-1} K x \subsetneq K$.

Proof. It suffices to prove that if $x^{-1} K x \subseteq K$, then some power of $x$ lies in $K$.

Fix a finite generating set $S$ for $K$ and a finite generating set $T$ for $H$ that contains $x$ and $S$. Fix $k \in K \backslash\{1\}$ and a geodesic word $v$ for $k$. Consider the rectangle in the Cayley graph of $H$ with sides labelled $x^{-n}, v, x^{n}, w^{-1}$ where $w$ is a geodesic word in the generators $S$. These sides are quasigeodesics in $H$ with constants depending only on $S, T$ and $x$. Such rectangles are uniformly thin, so for $n$ sufficiently large, there is a repetition in one of the following two lists of elements of $H$ (see the figures):

case $1:\left(x^{-i} k x^{i} \mid i \leq n\right)$,

case $2:\left(x^{i} w_{i} \mid i \leq n\right)$, where $w_{i}$ is a prefix of $w$ minimizing $d\left(x^{-i}, w_{i}\right)$.

In case 1 , we have $x^{-i} k x^{i}=x^{-j} k x^{j}$ for some $i \neq j$, and hence $x^{j-i}$ commutes with $k$. But $C_{H}(k)$ is a cyclic group that intersects $K$ nontrivially, so some power of $x$ lies in $K$.

In case 2 , we have $x^{i} w_{i}=x^{j} w_{j}$ for some $i \neq j$, and so $x^{j-i}=$ $w_{i} w_{j}^{-1} \in K$ as $w_{i} w_{j}^{-1}$ is a word in the generators $S$ of $K$. 


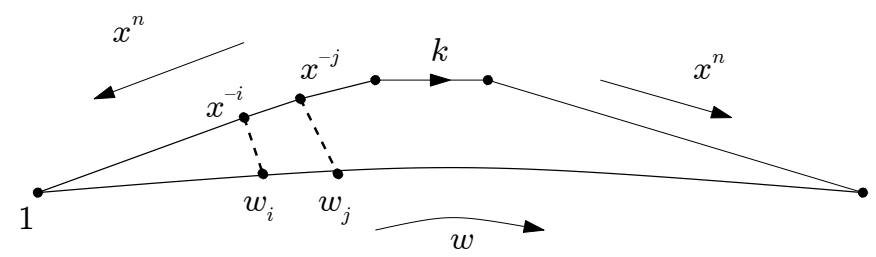

Lemma 4. Let $A$ be a group and let $K<A$ be a subgroup such that $K=\left\{a \in A \mid a^{-1} K a \subseteq K\right\}$. Let $\phi: K \rightarrow K$ be an automorphism and let $G=A *_{\phi}$ be the corresponding $H N N$ extension with stable letter $t$.

Then $N_{G}(K)=\langle K, t\rangle=K \rtimes_{\phi}\langle t\rangle$, and $C_{G}(K)=Z\left(K \rtimes_{\phi}\langle t\rangle\right)$, the centre of $K \rtimes_{\phi}\langle t\rangle$.

Proof. If $g \in G \backslash\langle K, t\rangle$ then, replacing $g$ by $g t^{i}$ for some $i \in \mathbb{Z}$ if necessary, we may assume that $g$ has a Britton normal form

$$
g=a_{0} t^{n_{1}} a_{1} \ldots t^{n_{\ell}} a_{\ell},
$$

where either $\ell>0$ and $n_{i} \neq 0, a_{i} \in A \backslash K$ for $1 \leq i \leq \ell$, or else $g=a_{0}=a_{\ell} \in A \backslash K$.

Fix $k \in K$ such that $a_{\ell} k a_{\ell}^{-1} \notin K$. Then

$$
a_{0} t^{n_{1}} a_{1} \ldots t^{n_{\ell}}\left(a_{\ell} k a_{\ell}^{-1}\right) t^{-n_{\ell}} \ldots a_{1}^{-1} t^{-n_{1}} a_{0}^{-1},
$$

is in normal form, and hence $g k g^{-1} \notin K$.

Proof of Theorem B. We maintain the notation established in the statement of the theorem. Lemma 2 tells us that $F=N_{H}(F)$, Lemma 3 tells us that $N_{H}(F)=\left\{x \in H \mid x^{-1} F x \subseteq F\right\}$, and Lemma 4 assures us that $C_{G}(F)=Z\left(F \rtimes_{\phi} \mathbb{Z}\right)$. It follows from these results that $C_{G}(F)=$ $\{1\}$ (and hence that $Z(G)=\{1\}$ ). Indeed, with the lemmas in hand, it is enough to note that a semidirect product $F \rtimes_{\phi} \mathbb{Z}$ has trivial centre if and only if $\phi \in \operatorname{Out}(F)$ has infinite order.

As an HNN extension of a torsion-free group, $G$ is torsion-free. Moreover, torsion-free hyperbolic groups have finite classifying spaces (see, e.g. [3, III.Г.3.21]), and hence so does any HNN extension amalgamating subgroups which themselves have finite classifying spaces (e.g. finitely generated free groups).

To complete the proof of the theorem, it only remains to prove that every injective homomorphism $\psi: G \rightarrow G$ is an inner automorphism. For this we invoke Bass-Serre theory. The subgroup $\psi(H)<G$ acts on the Bass-Serre tree for the HNN decomposition $G=H *_{\phi}$. The edge-stabilizers for this action are free and $H \cong \psi(H)$ does not split over a free group, so $\psi(H)$ fixes a vertex. The vertex stabilizers are the conjugates of $H$ in $G$, and hence $\psi(H) \subseteq g^{-1} H g$ for some $g \in G$. But $H$ is co-Hopfian [13], so in fact $\psi(H)=g^{-1} H g$. By composing with the inner automorphism $\operatorname{ad}_{g}$, we may assume that $\psi(H)=H$. Since $H$ is complete, $\left.\psi\right|_{H}=\left.\operatorname{ad}_{h}\right|_{H}$ for some $h \in H$, so composing with $\operatorname{ad}_{h}^{-1}$ we may assume that $\left.\psi\right|_{H}=\mathrm{id}_{H}$. 
Let $t$ be the stable letter of the HNN extension $G=H *_{\phi}$. Then $t^{-1} u t=\phi(u)$ for every $u \in F$. Applying $\psi$ we obtain $\psi(t)^{-1} u \psi(t)=$ $\phi(u)=t^{-1} u t$. Hence $\psi(t) t^{-1} \in C_{G}(F)=\{1\}$. Since $G=\langle H, t\rangle$, we deduce that $\psi$ (adjusted by inner automorphisms) is the identity map on $G$.

\section{REMARKS AND VARIATIONS}

(1). Theorem B remains valid if one replaces the hypothesis that $F<H$ is quasiconvex and free of rank 2 by the hypothesis that $F$ is free and malnormal (i.e. $x^{-1} F x \cap F=\{1\}$ for all $x \notin F$ ). Lemmas 1, 2, 3 become unnecessary with this alternative hypothesis.

(2). It is not essential that $F$ be free: instead, one can assume that $F$ is malnormal and belongs to a subgroup-closed class of groups $\mathcal{C}$ such that $H$ does not split over any group in $\mathcal{C}$.

(3). Regardless of whether one uses the original hypotheses on $F$ or one of the above alternatives (with $F \neq H$ ), one can replace the hypothesis that $\phi$ has infinite order in $\operatorname{Out}(F)$ by the hypothesis that $\phi$ has order $p>2$, say $\phi^{p}=\operatorname{ad}_{a}$. In this case, one can no longer conclude that $G$ is co-Hopfian but it will still be complete. Indeed, if $\psi: G \rightarrow G$ is a monomorphism, then following the proof of Theorem $\mathrm{B}$, one reduces to the situation where $\psi(H)=H$ and $\psi(t) t^{-1} \in C_{G}(F)$. But now $C_{G}(F)=\left\langle a t^{-p}\right\rangle$. If $\psi(t) t^{-1}=\left(a t^{-p}\right)^{r}$ for $r \neq 0$, then $\langle H, \psi(t)\rangle=$ $\left\langle H, t^{1-p r}\right\rangle$, and one sees that this is a proper subgroup of $G$ by observing that $G /\langle\langle H\rangle\rangle=\langle t\rangle \cong \mathbb{Z}$ and $|1-p r| \geq 2$.

(4). The reader may be dissatisfied with the fact that the inverting automorphism $\alpha: G \rightarrow G$ in our proof of Theorem A is inner. One can remedy this by taking a second, non-isomorphic, group $G^{\prime}$ of the type described in Theorem B, defining $\bar{\alpha}: G * G^{\prime} \rightarrow G * G^{\prime}$ to be the automorphism that restricts to $\alpha$ on $G$ and the identity on $G^{\prime}$.

(5). Miller and Schupp [12] prove that if $p$ and $q$ are integers and $G$ is a countable torsion-free group, then $G$ can be embedded in a complete hopfian group $G^{*}$ that has $p$ torsion and $q$ torsion, but has no $r$-torsion if $r$ is coprime to $p$ and $q$. If $G$ is finitely presented then so is $G^{*}$. Thus if $p$ and $q$ are odd and $G$ is a torsion-free group in which some element is conjugate to its inverse, for example the fundamental group of the Klein bottle, then $G^{*}$ will be a group of the type asked for by Hegarty and MacHale [6]. This begs the question of whether one might adapt the construction of [12] to ensure that $G^{*}$ is torsion-free if $G$ is. We shall return to this point in a future article [4]. 


\section{REFERENCES}

[1] J. Alonso, T. Brady, D. Cooper, V. Ferlini, M. Lustig, M. Mihalik, M. Shapiro, and H. Short, Notes on word hyperbolic groups, in Group Theory from a Geometrical Viewpoint, ed. by E. Ghys, A. Haefliger, and A. Verjovsky, World Scientific, 3-63, 1991

[2] M. Belolipetsky and A. Lubotzky, Finite groups and hyperbolic manifolds, Invent. math. 162, 459-472 (2005)

[3] M. R. Bridson and A. Haefliger, "Metric Spaces of Non-Positive Curvature", Grund. Math. Wiss. 319, Springer-Verlag, Heidelberg-Berlin, 1999.

[4] M.R. Bridson and H. Short, A complete embedding theorem for torsion-free groups, in preparation.

[5] M. Gromov, Hyperbolic groups, in "Essays in Group Theory" (S.M. Gersten, ed.), Springer-Verlag, MSRI Publ. 8 (1987), 75-263.

[6] P. Hegarty and D. MacHale, Minimal odd order automorphism groups, J. Group Theory 13 (2010), 243-255.

[7] H. Heineken and H. Liebeck, On p-groups with odd order automorphism groups, Arch. Math. (Basel) 24 (1973), 464-471.

[8] S. Kojima, Isometry transformations of hyperbolic 3-manifolds, Topology and its Appl. 29 (1988), 297-307.

[9] D.D. Long and A.W. Reid, On asymmetric hyperbolic manifolds, Proc.Camb.Phil.Soc 138 (2005), 301-306.

[10] W. Magnus, A. Karrass and D. Solitar, Combinatorial Group Theory: Presentations of groups in terms of generators and relations. Interscience Publishers [John Wiley \& Sons, Inc.], New York-London-Sydney, 1966.

[11] M.L. Mihalik and W. Towle, Quasiconvex subgroups of negatively curved groups, J. Pure and Appl. Alg. 95 (1994), 297-301.

[12] Charles F. Miller III and Paul E. Schupp, Embedding into Hopfian Groups, J. Alg., 17 (1971), 171-176.

[13] Z. Sela, Structure and rigidity in (Gromov) hyperbolic groups and discrete groups in rank 1 Lie groups II, Geom. Funct. Anal., 7 (1997), 561-593.

Martin R. Bridson, Mathematical Institute, University of Oxford, Andrew Wiles Building, Oxford OX2 6GG, U.K.

E-mail address: bridson@maths.ox.ac.uk

Hamish Short, Aix Marseille Université, CNRS, Centrale MarSEILle, I2M, UMR 7373, 13453, MARseille, France

E-mail address: hamish@cmi.univ-mrs.fr 